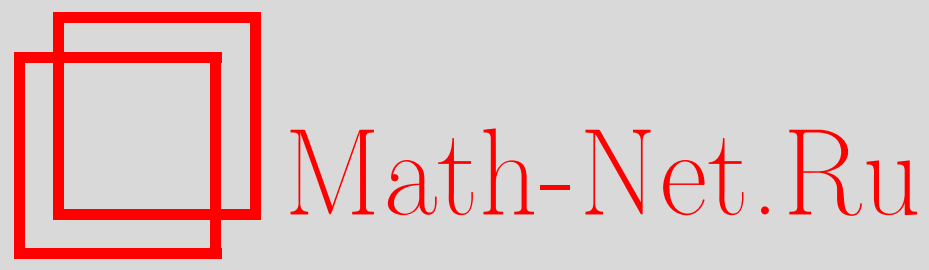

В. Ю. Калошин, Превалентность в пространствах конечногладких отображений, Функи. анализ и его прил., 1997, том 31, выпуск 2, 27-33

DOI: https://doi.org/10.4213/faa457

Использование Общероссийского математического портала MathNet.Ru подразумевает, что вы прочитали и согласны с пользовательским соглашением

http://www . mathnet.ru/rus/agreement

Параметры загрузки:

IP : 3.89.197.203

26 апреля 2023 г., 06:05:26

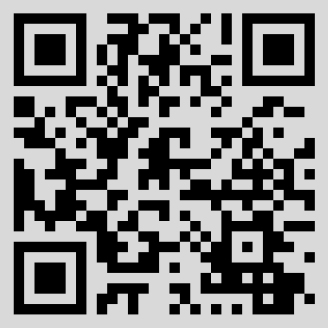


Функииональньй анализ и еәо приложения

1997, т. 31, вып. 2, с. 27-33

УДК $517.3,517.9$

\title{
Превалентность в пространствах конечногладких отображений
}

\author{
(c) 1997. В. Ю. КАлошин
}

В настоящей работе дано определение преобладающих («метрически превалирующих») множеств в нелинейных функциональных пространствах. Подмножество евклидова пространства мы называем метрически превалируюшим, если его дополнение имеет меру нуль. Не существует естественного способа обобщить определение «множества меры нуль» с конечномерных на бесконечномерные пространства [6]. Поэтому необходимо специально определить метрически превалирующее множество (множество полной меры) в бесконечномерном случае. Существует не один способ дать такое определение. Мы предлагаем один из возможных способов определить класс метрически превалирующих множеств в пространстве гладких отображений из гладкого компактного многообразия в гладкое. В статье показано, что класс метрически превалирующих множеств обладает рядом естественных свойств, в частности, пересечение конечного числа метрически превалирующих множеств является метрически превалирующим. Основным результатом данной работы является превалентный вариант теоремы Тома о трансверсальности.

По традиции, принятой в теории особенностей и теории динамических систем, свойство присуще «почти всем» отображениям (или потокам), если оно присуще множеству, содержащему счетное пересечение открытых всюду плотных подмножеств в соответствующем функциональном пространстве и называемому массивным. Однако даже в конечномерных пространствах такое множество может иметь сколь угодно малую меру Лебега например равную нулю. В настоящей статье доказано, что теорема Тома о трансверсальности справедлива для существенно более «густого», чем просто массивное, множества. Естественно пересмотреть с точки зрения превалентности другие классические результаты из теории особенностей и теории динамических систем, такие, как мультиструйная теорема трансверсальности, теорема Мазера (Mather) об устойчивости, теорема Купки-Смейла для динамических систем и другие, и это сделано в статье [9]. Понятие превалентности в линейных банаховых пространствах введено и исследовано в [8]. Один из путей определить класс превалирующих множеств в пространствах гладких отображений многообразий, существенно отличающийся от представленного в данной статье, предложен в [7].

Определения типичности, использующие меру Лебега в конечномерном пространстве, предлагались А. Н. Колмогоровым [10] и В. И. Арнольдом [11]. Эти определения цитируются и обсуждаются в статье [9]. Здесь укажем только, что конечномерный аналог определения Арнольда допускает в качестве превалент- 
ных множества сколь угодно малой меры. Конечномерный аналог понятия превалентности, определенного в этой статье, допускает в качестве превалентных только множества полной меры. Наше определение модифицирует определение Арнольда.

Автор выражает признательность Ю. С. Ильяшенко за постоянное внимание к работе и полезные обсуждения, а также Р. И. Богданову за помощь при подготовке рукописи.

\section{§1. Формулировка основного результата}

Пусть $C^{k}(M, N)$ - пространство отображений из гладкого компактного многообразия $M$ в гладкое многообразие $N$ конечной гладкости $k$ с однородной $C^{k}$-топологией, а $J^{m}(M, N)$ - пространство $m$-струйных расширений отображений из $C^{k}(M, N)$, где $m<k$.

Обозначим через $B^{n}$ единичный шар в $\mathbb{R}^{n}$ с центром в нуле. Рассмотрим пространство $C^{k}\left(M \times B^{n}, N\right)$ с $C^{k}$-топологией как пространство $n$-параметрических семейств $\left\{f_{\varepsilon}\right\}_{\varepsilon \in B^{n}}$ отображений из пространства $C^{k}(M, N), C^{k}$-гладко зависящих от параметра.

ОПРЕДЕЛЕНИЕ 1. Назовем множество $P \subset C^{k}(M, N)$ метрически превалируюшим, если существует целое положительное $n$, такое, что для любого отображения $f \in C^{k}(M, N)$ существует $n$-параметрическое семейство $\left\{f_{\varepsilon}\right\}_{\varepsilon \in B^{n}} \in$ $C^{k}\left(M \times B^{n}, N\right), f_{0}=f$, которое удовлетворяет следующему условию: для каждого $n$-параметрического семейства $\left\{g_{\varepsilon}\right\}_{\varepsilon \in B^{n}}$, близкого к $\left\{f_{\varepsilon}\right\}_{\varepsilon \in B^{n}}$ в $C^{k}\left(M \times B^{n}, N\right)$, выполнено условие

$$
\operatorname{mes}\left\{\varepsilon \in B^{n}: g_{\varepsilon} \notin P\right\}=0 \text {, }
$$

где mes есть $n$-мерная мера Лебега.

ОПРЕДЕЛЕНИЕ 2. Множество, дополнение к которому является метрически превалирующим, назовем метрически пренебрежимым.

Пусть $K$ - подмногообразие в пространстве струй $J^{m}(M, N)$. Множество отображений из $C^{k}(M, N), k>m, m$-струйные расширения которых трансверсальны $K$, обозначим через $\operatorname{Thom}_{m}(K)$.

ПРЕВАЛЕНТНАЯ ТЕОРЕМА ТРАНСВЕРСАЛЬНОСТИ. Множество отображений $\operatorname{Thom}_{m}(K) \subset C^{k}(M, N)$ является метрически превалируюшим для любого $k>m$.

\section{Свойства метрически превалирующих множеств}

Свойство 1. а) Любое подмножество метрически пренебрежимого множества метрически пренебрежимо.

б) Объединение любого, может быть бесконечного, числа метрически превалирующих множеств является метрически превалирующим.

Свойство 2. Метрически превалирующее множество является всюду плотным.

Свойство 3. Пересечение любого конечного числа метрически превалирующих множеств является метрически превалирующим. 
Свойства 1 и 2 следуют непосредственно из определений 1 и 2. Свойство 3 будет доказано в $\S 4$.

\section{§2. Доказательство превалентной теоремы трансверсальности}

Фиксируем произвольное отображение $f \in C^{k}(M, N)$ и некоторую его окрестность $U_{f}$ в $C^{k}$-топологии. Пусть $K$ - подмногообразие в пространстве $m$-струй $J^{m}(M, N)$ и $(V, \varphi)$ - координатная окрестность на $M$. Обозначим множество отображений $g$ из $U_{f}$, ограничение которых на $V$ трансверсально $K$, т. е. $j_{x}^{m} g$ трансверсально $K$ для любой точки $x \in V$, через $\operatorname{Thom}_{f}(K, V)$.

Ввиду свойства 3 и того, что многообразие $M$ компактно, достаточно доказать, что множество $\operatorname{Thom}_{f}(K, V)$ является метрически превалирующим для любой координатной окрестности $V$. Действительно, если $\bigcup_{i} V_{i}-$ некоторое покрытие многообразия $M$, то ввиду компактности можно выделить из него конечное подпокрытие, $\bigcup_{i=1}^{d} V_{i}=M$, и воспользоваться очевидным соотношением

$$
\operatorname{Thom}_{m}(K) \cap U_{f}=\bigcap_{i=1}^{d} \operatorname{Thom}_{f}\left(K, V_{i}\right) .
$$

ЛОКАЛЬНАЯ ПРЕВАЛЕНТНАЯ ТЕОРЕМА ТРАНСВЕРСАЛЬНОСТИ. Во введенных выше обозначениях множество $\operatorname{Thom}_{f}(K, V) \subset C^{k}(M, N)$ является метрически превалируюшим для любой координатной окрестности $V$.

ДоказАтЕльство. Докажем две вспомогательные леммы.

ЛЕмма 1. Пусть имеется непрерьвно дифферениируемое отображение $F: M \times B \rightarrow N_{0}$, где $M, N_{0}$ u $B$ - гладкие многообразия, причем $\operatorname{dim} M<$ $\operatorname{dim} B$. Рассмотрим его как семейство отображений, зависящих от параметра, $F_{\varepsilon}: M \rightarrow N_{0}$. Тогда если отображение $F$ трансверсально подмногообразию $K$ многообразия $N_{0}$, то для почти всех (по мере) значений параметра $F_{\varepsilon}: M \rightarrow N_{0}$ трансверсально $K$, или

$$
\operatorname{mes}\left\{\varepsilon \in B: F_{\varepsilon} \notin P\right\}=0 \text {. }
$$

ДокАЗАТЕльство. Обозначим через $D_{(x, \varepsilon)} F$ дифференциал отображения $F$ в точке $(x, \varepsilon)$. По определению отображение $F$ трансверсально многообразию $K$, если выполнено условие

$$
D_{(x, \varepsilon)} F\left(T_{(x, \varepsilon)}(M \times B)\right)+T_{F(x, \varepsilon)} K=T_{F(x, \varepsilon)} N_{0}
$$

для всех точек $(x, \varepsilon)$ из прообраза $F^{-1}(K) \subset M \times B$. Из теоремы о неявной функции следует, что множество $\widehat{K}=F^{-1}(K)$ является $C^{1}$-гладким подмногообразием в $M \times B$. Рассмотрим ограничение проекции $M \times B \rightarrow B$ вдоль $M$ на $\widehat{K}$, которое обозначим через $\pi_{K}: \widehat{K} \rightarrow B$. Напомним конечногладкий вариант теоремы Сарда: мера множества критических значений всякого $C^{1}$-гладкого отображения многообразий $f: A \rightarrow B$ равна нулю при условии, ито $\operatorname{dim} A<2 \operatorname{dim} B$ (см., например, [2, с. 88-89]). Заметим, что ввиду неравенства $\operatorname{dim} \widehat{K}<\operatorname{dim} M+\operatorname{dim} B<2 \operatorname{dim} B$ к отображению $\pi_{K}: \widehat{K} \rightarrow B$ 
можно применить эту теорему, из которой следует, что для почти всех значений параметра

$$
D_{(x, \varepsilon)} F\left(T_{(x, \varepsilon)} \widehat{K}\right)+D_{(x, \varepsilon)} F\left(T_{(x, \varepsilon)} M\right)=D_{(x, \varepsilon)} F\left(T_{(x, \varepsilon)}(M \times B)\right),
$$

HO

$$
D_{(x, \varepsilon)} F\left(T_{(x, \varepsilon)}(M \times B)\right)+T_{F(x, \varepsilon)} K=D_{(x, \varepsilon)} F\left(T_{(x, \varepsilon)} M\right)+T_{F(x, \varepsilon)} K=T_{F(x, \varepsilon)} N_{0} .
$$

Лемма доказана.

Из классической литературы (см. [2-4]) известно, что если рассмотреть $C^{k}$-гладкое отображение $F$ из компактной области $U \subset \mathbb{R}^{q}$ в $\mathbb{R}^{p}$ и зафиксировать системы координат в $\mathbb{R}^{p}$ и $\mathbb{R}^{q}$, то существует «универсальное» (подходящее для любого $F$ ) возмущение вида $F_{\varepsilon}(x)=F(x)+\sum_{i=1}^{n} \varepsilon_{i} x_{i}$, где $x_{1}, \ldots, x_{n}$ всевозможные мономы степени не выше $m$ от координат точки $x \in U$, умноженные на базисные векторы из $\mathbb{R}^{p}$, такое, что у отображения $\varphi:(x, \varepsilon) \rightarrow\left(j_{x}^{m} F_{\varepsilon}\right)$ нет критических значений, а значит, $\varphi$ трансверсально любому подмногообразию пространства $m$-струйных расширений $J^{m}\left(U, \mathbb{R}^{p}\right)$.

Лемма 2. Во введенных выше обозначениях для всех достаточно близких $\kappa\left\{F_{\varepsilon}\right\}_{\varepsilon \in B^{n}}$ n-параметрических семейств $\left\{G_{\varepsilon}\right\}_{\varepsilon \in B^{n}}$ выполнено свойство регулярности, а именно отображение $\varphi_{G}:(x, \varepsilon) \rightarrow\left(j_{x}^{m} G_{\varepsilon}\right)$ не имеет критических значений.

ДоказАтельство. Нетрудно видеть, что $\varphi:(x, \varepsilon) \rightarrow\left(j_{x}^{m} F_{\varepsilon}\right)$ - отображение пространств одинаковой размерности. Регулярность означает, что якобиан этого отображения - непрерывная отличная от нуля функция на компакте $U$. Это свойство, очевидно, сохраняется при малых $C^{1}$-возмущениях. Поэтому якобиан отображения $\varphi_{G}:(x, \varepsilon) \rightarrow\left(j_{x}^{m} G_{\varepsilon}\right)$ также отделен от нуля. Лемма доказана.

Пусть $U_{f}$ - некоторая достаточно малая окрестность отображения $f$ в $C^{k}(M, N)$. Без ограничения общности можно считать, что для любой точки $p \in M$ существует пара координатных окрестностей $(\widehat{V}, \eta)$ и $(W, \psi)$ на многообразиях $M$ и $N$ соответственно, таких, что

(a) $V$ - окрестность точки $p$ на многообразии $M$ и $V \subset \widehat{V}$;

(б) множество образов точек из $\widehat{V}$ под действием отображений из $U_{f}$ содержится в карте $W$, т.е. $U_{f}(V) \subset W$.

Пусть $\rho: M \rightarrow[0,1]$ - функция класса $C^{\infty}$, такая, что

$$
\rho(p)= \begin{cases}1 & \text { при } p \text { из замыкания окрестности } V, \\ 0 & \text { при } p \text { из дополнения к } \widehat{V} \text {. }\end{cases}
$$

Определим семейство отображений $\left\{f_{\varepsilon}\right\}_{\varepsilon \in B^{n}}$ формулой

$$
f_{\varepsilon}(p)=\left\{\begin{array}{l}
\psi^{-1}\left(\psi(f(p))+\rho(p)\left(\sum_{i=1}^{n} \varepsilon_{i} x_{i}(\psi(f(p)))\right)\right) \text { при } x \text { из } \widehat{V}, \\
f(p) \text { при } p \text { из дополнения к } \widehat{V} .
\end{array}\right.
$$

Из явного вида семейств $\left\{f_{\varepsilon}\right\}_{\varepsilon \in B^{n}}$ следует, что $\left\{f_{\varepsilon}\right\}_{\varepsilon \in B^{n}} \in C^{k}\left(M \times B^{n}, N\right)$, т. е. это семейство $C^{k}$-гладко зависит от параметра. 
ЗАмЕЧАНИЕ. В формулу (6) вместо отображения $f$ можно подставить любое отображение $g$ из $U_{f}$.

Из лемм 1 и 2 следует, что множество отображений $\operatorname{Thom}_{f}(K, V)$ является метрически превалируюшим. Действительно, пусть $N_{0}=J^{m}(M, N), B=B^{n}$, $U=\varphi(\widehat{V})$ и $F_{\varepsilon}=\psi\left(f_{\varepsilon}\left(\varphi^{-1}(x)\right)\right)$; тогда последовательное применение лемм 1 и 2 завершает доказательство локальной превалентной теоремы трансверсальности.

\section{§3. Локальная линейная структура в пространстве отображений многообразий}

ЛЕмма 3. В окрестности каждой точки пространство $C^{k}$-гладких отображений из гладкого компактного многообразия $M$ в гладкое многообразие $N$ можно ввести локальную линейную структуру.

ЗАмЕЧАНИЕ. Лемма 3 необходима для доказательства свойства 3.

ДокАЗАтЕльство. Фиксируем произвольное отображение $f \in C^{k}(M, N)$. Без ограничения общности можно считать, что $N$ является подмногообразием в $\mathbb{R}^{r}$, где $r=2 \operatorname{dim} N+1$. В каждой точке $y \in N$ проведем касательную плоскость $T_{y} N$. Это аффинное подпространство в $\mathbb{R}^{r}$. Выбор точки $y$ как начала координат превращает это пространство в линейное с естественной структурой касательного пространства к $N$.

Для каждой точки $y \in N$ существует окрестность $W_{y}$ на $N$, ортогональная проекция которой на касательное пространство $T_{y} N$ является диффеоморфизмом. Обозначим этот диффеоморфизм через $\pi_{y}$. Окрестность $W_{y}$ можно выбрать так, чтобы для некоторого положительного $\rho$ было выполнено условие $\{z \in N:|y-z|<\rho\} \subset W_{y}$. Для каждого отображения $f$ из $C^{k}(M, N)$ определим окрестность $U_{f}$ следующим образом:

$$
U_{f}=\left\{g \in C^{k}(M, N): \max _{x \in M}|g(x)-f(x)|<\rho / 2\right\} .
$$

Введем операцию сложения + для произвольных отображений $g_{1}, g_{2}$ из $U_{f}$ так:

$$
g_{1}+g_{2}: M \rightarrow N, \quad x \rightarrow \pi_{f(x)}^{-1}\left(\pi_{f(x)} g_{1}(x)+\pi_{f(x)} g_{2}(x)\right) .
$$

Если определить операцию умножения на константу для $g \in U_{f}$ формулой

$$
(\lambda g)(x)=\pi_{f(x)}^{-1}\left(\lambda \pi_{f(x)} g\right)(x),
$$

то в некоторой окрестности $U_{f}$ произвольного отображения $f \in C^{k}(M, N)$ имеется локальная линейная структура. Лемма доказана.

ЗАмЕчАНИЕ. Используя доказательство леммы 3, нетрудно показать, что пространство $C^{k}(M, N)$ является банаховым многообразием.

\section{§4. Доказательство свойства 3}

Достаточно доказать, что пересечение двух метрически превалирующих множеств является метрически превалирующим. В случае пересечения конечного числа, большего двух, таких множеств можно применить индукцию по числу множеств. 
Допустим, что $P_{1}$ и $P_{2}$ - метрически превалирующие множества. Фиксируем произвольное отображение $f \in C^{k}(M, N)$. По определению 1 существуют $n_{i}$-параметрические семейства $\left\{f_{\varepsilon_{i}}\right\}_{\varepsilon_{i} \in B^{n_{i}}}$, такие, что выполнено условие

$$
\operatorname{mes}_{i}\left\{\varepsilon_{i} \in B^{n_{i}}: f_{\varepsilon_{i}} \notin P_{i}\right\}=0,
$$

где mes $_{i}$ есть $n_{i}$-мерная мера Лебега для $i=1,2$.

Пусть $n=n_{1}+n_{2}$. Достаточно доказать существование $n$-параметрического семейства $\left\{f_{\left(\varepsilon_{1}, \varepsilon_{2}\right)}\right\}_{\left(\varepsilon_{1}, \varepsilon_{2}\right) \in B^{n}}$, такого, что для любого достаточно близкого $\mathrm{K}$ нему $n$-параметрического семейства $\left\{g_{\left(\varepsilon_{1}, \varepsilon_{2}\right)}\right\}_{\left(\varepsilon_{1}, \varepsilon_{2}\right) \in B^{n}}$ в $C^{k}\left(M \times B^{n}, N\right)$ выполнено условие

$$
\operatorname{mes}\left\{\left(\varepsilon_{1}, \varepsilon_{2}\right) \in B^{n}: f_{\left(\varepsilon_{1}, \varepsilon_{2}\right)} \notin P_{1} \cap P_{2}\right\}=0 .
$$

Без ограничения общности можно считать, что семейства отображений $\left\{f_{\varepsilon_{i}}\right\}_{e_{i} \in B^{n_{i}}}(i=1,2)$ лежат в некоторой окрестности отображения $f$, а значит, определена операция сложения + . Определим $n$-параметрическое семейство $\left\{f_{\left(\varepsilon_{1}, \varepsilon_{2}\right)}\right\}_{\left(\varepsilon_{1}, \varepsilon_{2}\right) \in B^{n}}$ следующим образом (см. формулу $\left.(8)\right)$ :

$$
f_{\left(\varepsilon_{1}, \varepsilon_{2}\right)}(x)=f_{\varepsilon_{1}}+f_{\varepsilon_{2}}=\pi_{f(x)}^{-1}\left(\pi_{f(x)} f_{\varepsilon_{1}}(x)+\pi_{f(x)} f_{\varepsilon_{2}}(x)\right)
$$

для любого $x \in M$. Нетрудно убедиться, что выполнено неравенство

$$
\begin{aligned}
& \operatorname{mes}\left\{\left(\varepsilon_{1}, \varepsilon_{2}\right) \in B^{n}: f_{\left(\varepsilon_{1}, \varepsilon_{2}\right)} \notin P_{1} \cap P_{2}\right\} \\
& \leqslant \operatorname{mes}\left\{\left(\varepsilon_{1}, \varepsilon_{2}\right) \in B^{n}: f_{\left(\varepsilon_{1}, \varepsilon_{2}\right)} \notin P_{1}\right\}+\operatorname{mes}\left\{\left(\varepsilon_{1}, \varepsilon_{2}\right) \in B^{n}: f_{\left(\varepsilon_{1}, \varepsilon_{2}\right)} \notin P_{2}\right\} .
\end{aligned}
$$

Пусть $g_{\varepsilon_{2}}=f_{\left(\varepsilon_{01}, \varepsilon_{2}\right)}\left(g_{\varepsilon_{1}}=f_{\left(\varepsilon_{1}, \varepsilon_{02}\right)}\right)$. Из явного вида формулы $(12)$ и определения 1 , которое позволяет «шевелить» семейство $\left\{f_{\varepsilon_{i}}\right\}_{\varepsilon_{i} \in B^{n_{i}}}(i=1,2)$ с сохранением формулы (1), следует, что после соответствующей перепараметризации (уменьшения базы параметров) будут выполнены условия

$$
\left\{\varepsilon_{0 i} \in B^{n_{i}}: \operatorname{mes}\left\{\varepsilon_{\hat{i}} \in B^{n_{\hat{i}}}: g_{\varepsilon_{\hat{i}}} \notin P_{\hat{i}}\right\} \neq 0\right\}=\varnothing
$$

для любых значений параметров $\left(\varepsilon_{0 i}, \varepsilon_{\hat{i}}\right) \in B^{n_{i}} \times B^{n_{\hat{i}}}$, где $\{i, \hat{i}\}=\{1,2\}$. Тогда по теореме Фубини

$$
\operatorname{mes}\left\{\left(\varepsilon_{1}, \varepsilon_{2}\right) \in B^{n}=B^{n_{1}} \times B^{n_{2}}: f_{\left(\varepsilon_{1}, \varepsilon_{2}\right)} \notin P_{1} \cap P_{2}\right\}=0 .
$$

Свойство доказано.

\section{ЛитеРАтУРА}

1. Аносов Д. В. Гладкие динамические системы. Итоги науки и техники. Современные проблемы математики. Фундаментальные направления, ВИНИТИ, т. 1 , c. $149-242$.

2. Арнольд В. И. Дополнительные главы теории обыкновенных дифференциальных уравнений. Наука, 1978.

3. Арнольд В. И., Варченко А. Н., Гусейн-Заде С. М. Особенности дифференцируемых отображений. Наука, 1982.

4. Голубичкий М., Гийемин В. Устойчивые отображения и их особенности, Мир, 1977. 
5. Палис Ж., Ди Мелу В. Геометрическая теория динамических систем. Мир, 1986.

6. Судаков В. Н. Линейные множества с квазиинвариантной мерой. ДАН СССР, 127, 524-525 (1959).

7. Tsujii $M$. A measure on the space of smooth mappings and dynamical system theory. J. Math. Soc. Jpn, 44, No. 3, 415-425 (1992).

8. Hunt B., Sauer T., Yorke J. A. Prevalence: a translation invariant almost every for infinite dimensional spaces. Bull. Am. Math. Soc., 27, 217-238 (1992); Prevalence: an addendum, 28, 306-307 (1993).

9. Калошин В. Ю. Некоторые превалентные свойства гладких динамических систем. В сб.: Дифференциальные уравнения с вещественным и комплексным временем, Труды матем. инст. им. В. А. Стеклова, 213, 123-151 (1996).

10. Колмогоров А. Н. Общая теория динамических систем и классическая механика. В сб.: Международный математический конгресс в Амстердаме, Физматлит, Москва, 1961, с. 187-208.

11. Арнольд В. И. Малые знаменатели І. Об отображениях окружности на себя. Изв. АН СССР, сер. матем., 25, 21-86 (1961).

Московский государственный университет, механико-математический факультет
Поступило в редакцию 30 марта 1996 г. 